\title{
Patient Satisfaction Questionnaires in Trainee Evaluations
}

\author{
Dieneke Hubbeling • David Chang
}

Received: 17 September 2013 / Accepted: 21 November 2013 /Published online: 15 January 2014

(C) Academic Psychiatry 2014

To the editor:

Setting up appropriate assessments for postgraduate trainees in psychiatry is difficult. Members of the public, i.e. possible future patients, need to be sure that somebody who is recognized as a psychiatrist is able to function as such. However, it is less clear how one should assess this. Brittlebank et al. [1] added to this discussion by describing a study of different types of workplace-based assessments, for example a mini-assessed clinical encounter, whereby a supervisor observes the trainee performing a specific task such as assessing suicide risk and gives feedback afterwards. They found correlations between most measures, for example the correlation between case-based discussion and mini-assessed clinical encounter was $0.61(p<$ 0.001). However, the patient satisfaction questionnaire results seemed to be different and did not correlate with other measures.

Brittlebank et al. [1] are not the first to suggest that patient satisfaction questionnaires appear to measure something different from other educational assessments. Whelan et al. [2] found only a moderate correlation between the communication skills scores of the simulated patient (i.e. actor) and the overall score of the assessing psychiatrist, namely 0.41 (95\% confidence interval, 0.35-0.48). Mellsop et al. [3] asked observing psychiatrists and patients exactly the same questions about trainees' communication skills and found mostly low correlations which were not statistically significant. The only significant correlation was negative $(-0.441, p=0.01)$ for asking appropriate questions. Explanations for these findings are necessary and Brittlebank et al. rightly argue for this.

These findings have two possible explanations. First, there is some evidence that satisfaction with treatment is partly determined by the patient's personality [4], something that

D. Hubbeling $(\bowtie) \cdot$ D. Chang

Springfield University Hospital, London, UK

e-mail: dieneke@doctors.org.uk certainly needs further study if one wants to use patient satisfaction questionnaires to evaluate the skills of trainee psychiatrists and to assess the performance of the trainees and not the personality of the patient.

Second, lack of correlation might be because patient satisfaction scores tend to be high. Brittlebank et al. [1] reported that of all the measurements, the patient satisfaction questionnaire had the highest average score, namely 5.32 on a 6-point Likert scale, and the lowest SD of 0.50 . Therefore, the vast majority of patients thought that trainee psychiatrists were very good or excellent. It is quite likely that candidates who scored high on the other measures scored similar on patient satisfaction as candidates who scored low, which can explain the lack of correlation.

Brittlebank et al. [1] stated that it is important to include different viewpoints in the assessment of trainees, and we agree. However, it will be necessary to investigate the patient satisfaction questionnaire more carefully, and check whether responses correlate with other patient-related factors, not only with trainee factors. One should also try to develop a measure of patient satisfaction that is less skewed, although this might be difficult.

\section{References}

1. Brittlebank A, Archer J, Longson D, Malik A, Bhugra DK. Workplace-based assessments in psychiatry: evaluation of a whole assessment system. Acad Psychiatry. 2013;37(5):301-7.

2. Whelan P, Church L, Kadry K. Using standardized patients' marks in scoring postgraduate psychiatry OSCEs. Acad Psychiatry. 2009;33(4): 319-22.

3. Mellsop GW, MacDonald J, El Badri S, Menkes D. Patients' appraisal of psychiatric trainee interview skills. Acad Psychiatry. 2012;36(5): 374-9.

4. Green SM, Hadjistavropoulos T, Sharpe D. Client personality characteristics predict satisfaction with cognitive behavior therapy. J Clin Psychol. 2008;64(1):40-51. 